\title{
Factors Influencing the Adequacy of Bowel Preparation in Patients With Developmental Disabilities
}

\author{
Jose Mari Parungao ${ }^{\mathrm{a}, \mathrm{f}, \mathrm{g}}$, Charina Reyes ${ }^{\mathrm{b}, \mathrm{f}}$, Nancy Jackson ${ }^{\mathrm{c}}$, \\ Nancy Roizen ${ }^{\mathrm{d}}$, Michael Piper ${ }^{\mathrm{e}}$
}

\begin{abstract}
Background: The rate of inadequate bowel preparation in the general population is approximately $23 \%$. As more individuals with developmental disabilities enter late adulthood, a concomitant rise in endoscopic procedures for this population, including screening colonoscopies, is anticipated. However, there are sparse data on the adequacy of bowel preparation in patients with developmental disabilities.
\end{abstract}

Methods: A retrospective analysis of 91 patients with developmental disabilities who underwent colonoscopy from 2006 to 2014 was performed. Bowel preparation adequacy from these procedures was evaluated, together with other data, including age, developmental disability diagnoses, procedure type, indication and setting.

Results: Mean age at the time of endoscopy was $52.6 \pm 13.4$ years, with an age range of 18 - 74 years. Inadequate bowel preparation was found in approximately $51 \%$ of documented cases. Outpatients were more likely to have adequate bowel preparation compared to inpatients, with an odds ratio of 2.75 (95\% confidence interval: 1.14 - 6.62, $\mathrm{P}=0.022)$. No other major factors identified had any statistically significant influence on the adequacy of bowel preparation.

Conclusion: Over half of patients with developmental disabilities undergoing colonoscopy had inadequate bowel preparations in our study, which is more than twice the rate for the general population. Furthermore, outpatients were 2.75 times more likely to have adequate bowel preparation compared to inpatients. Further studies are recommended to improve endoscopic practices for this patient population.

Manuscript submitted November 20, 2018, accepted December 4, 2018

aUnited Medical Center, Washington, DC 20032, USA

bivision of Developmental-Behavioral Pediatrics, University of Maryland Medical Center, Baltimore, MD 21201, USA

'Department of Research, Providence-Providence Park Hospital, Southfield, MI 48075, USA

${ }^{\mathrm{d} D i v i s i o n}$ of Developmental-Behavioral Pediatrics and Psychology, Rainbow Babies and Children's Hospital, Cleveland, OH 44106, USA

eDepartment of Gastroenterology, Providence-Providence Park Hospital, Southfield, MI 48075, USA

fThese authors contributed equally to this work.

gCorresponding Author: Jose Mari Parungao, United Medical Center, 1310

Southern Ave SE, Washington, DC 20032, USA.

Email: JParungao@united-medicalcenter.com

doi: https://doi.org/10.14740/gr1118
Keywords: Developmental disability; Endoscopy; Bowel preparation

\section{Introduction}

The Centers for Disease Control and Prevention (CDC) estimates that the prevalence of children with developmental disabilities has increased by $17.1 \%$ over the past 12 years [1], with longer life expectancies than previously projected $[2,3]$. With the rising number of individuals with developmental disabilities entering late adulthood, a concomitant rise in the number of endoscopic procedures, including screening colonoscopies, is anticipated. Compared to the general population, individuals with developmental disabilities present with higher rates of gastrointestinal disorders, such as celiac disease and bowel dysfunction $[4,5]$. Despite the increased prevalence of gastrointestinal disorders in this population, individuals with developmental disabilities experience a number of health disparities associated with multiple factors, including lack of access to quality healthcare, inadequate healthcare provider education to meet their unique needs and poor access to preventative measures [6]. Literature suggests that these patients are less likely to participate in colorectal cancer screening compared to the general population, with an absolute difference of $15.2 \%$ [7].

In order to achieve a high-quality and effective colonoscopy, an adequate bowel preparation must be achieved [8]. Inadequate bowel preparation may result in failure to identify adenomas and other high-risk lesions $[9,10]$. In addition, incomplete or suboptimal bowel preparation comes at a considerable cost to healthcare systems [11]. Current literature estimates that the rate of inadequate bowel preparation in colonoscopies is approximately $23.1 \%$ [9]. Adults with intellectual and developmental disabilities, in particular, have comorbidities such as an increased risk for gastrointestinal dysmotility that may affect preparation and compliance, and thus can impact the quality of the procedure [12]. There is a current paucity of literature regarding bowel preparation in patients with developmental disabilities.

The purpose of this study was to determine the rate of, and factors related to, adequate bowel preparation in lower endoscopic procedures for patients with developmental disabilities, specifically autism spectrum disorder, intellectual disability and Down syndrome. We hypothesized that there would be a 
Table 1. Developmental Disabilities Categorization

\begin{tabular}{ll}
\hline General categories & Diagnoses by ICD-9 coding \\
\hline Autism Spectrum Disorder & Autistic Disorder, Current or Active State \\
& Asperger Syndrome \\
Developmental Delay NOS & Other Pervasive Developmental Disorder \\
Down Syndrome & Developmental Delay NOS \\
Intellectual Disability & Down Syndrome \\
& Intellectual Disasbility NOS \\
& Mild Intellectual Disability \\
& Moderate Intellectual Disability \\
& Severe Intellectual Disability \\
\hline
\end{tabular}

ICD: International Classification of Diseases; NOS: not otherwise specified.

lower overall rate of adequate bowel preparation in this patient group due to the unique challenges faced by these individuals, compared to the general population based on current estimates in the literature. We also hypothesized that individuals with developmental disabilities would have better rates of bowel preparation in the inpatient setting, due to more optimal hospital support in terms of purge administration, compared to their outpatient counterparts.

\section{Patients and Methods}

A retrospective analysis of consecutive patients with developmental disabilities who underwent colonoscopy in four in-hospital endoscopic centers from August 2006 to September 2014 was performed. The study was approved by the institutional review board, and due to its retrospective nature, a waiver of informed consent was obtained.

Data collection was initiated by searching our electronic database, using International Classification of Diseases 9 (ICD9) codes to capture the patient population of interest to our study. These were then generalized into broader categories for data analysis as outlined in Table 1 . The four main categories were: 1) Autism Spectrum Disorder (ASD); 2) Developmental Delay not otherwise specified (NOS); 3) Down Syndrome; and 4) Intellectual Disability. The patients under the categories of Autism and Down Syndrome included those with and without accompanying intellectual disability, and therefore, those without these associated diagnoses were classified under the Intellectual Disability category. This list was then cross-referenced with the respective Current Procedure Terminology (CPT) codes for all lower endoscopic procedures to arrive at the final data set for analysis (Table 2). The investigators reviewed the individual electronic charts and procedure reports manually.

Patient data collected from the electronic chart included the following variables: age at the time of procedure, developmental disability diagnoses, procedure type and indication (i.e. diagnostic or therapeutic) and the procedure setting (i.e. inpatient or outpatient).

Bowel preparation was classified as either adequate or inadequate. Adequate bowel preparation was defined as those reported as excellent, good or adequate. Conversely, inadequate bowel preparation was defined as those reported as suboptimal, fair, inadequate or poor. This classification is consistent with prior literature that evaluated bowel preparation adequacy [13]. The bowel preparation classifications are assessments made by individual endoscopists at the time of the procedure using the Aronchick scale, as documented in the medical record [14]. These assessments were also consistent with the electronic prompts provided by the endoscopic procedure documentation software where these procedures were performed, where available.

\section{Statistical analysis}

Statistical analyses were performed using IBM SPSS Statistics Version 23.0. Fisher's exact tests and Pearson $\chi^{2}$ analyses were used for non-random associations between two independent groups and other identified categorical variables. Student's $t$ test was used to identify differences in continuous variables, such as age, between the two groups of patients with adequate or inadequate bowel preparations. Data obtained from Fisher's exact tests and $\chi^{2}$ analyses were also used to calculate for odds ratios after identifying significant differences using univariate analysis. A P value less than 0.05 was considered to be statistically significant.

\section{Results}

From 2006 to 2014 , a total of 150 patients with developmental

Table 2. Current Procedure Terminology (CPT) Codes Used for Database Search

\begin{tabular}{ll}
\hline Procedure & CPT code/s \\
\hline Ileoscopy/pouchoscopy & $44380-44386$ \\
Flexible sigmoidoscopy & $45330-45345$ \\
Colonoscopy through stoma & $44387-44397$ \\
Colonoscopy & $45378-45392$ \\
\hline
\end{tabular}


Table 3. Factors Affecting Bowel Preparation Adequacy

\begin{tabular}{|c|c|c|c|}
\hline & Adequate prep $(n=45)$ & Inadequate prep $(n=46)$ & P value \\
\hline Age (mean $\pm \mathrm{SD}$, in years) & $50 \pm 13$ & $55 \pm 13$ & 0.491 \\
\hline \multicolumn{4}{|l|}{ Location } \\
\hline Inpatient & $12(34 \%)$ & $23(66 \%)$ & \\
\hline \multicolumn{4}{|l|}{ Indication } \\
\hline Therapeutic & $29(51 \%)$ & $27(49 \%)$ & \\
\hline \multicolumn{4}{|l|}{ Time of procedure } \\
\hline Morning & $27(57 \%)$ & $20(43 \%)$ & 0.643 \\
\hline Afternoon & $15(51 \%)$ & $14(48 \%)$ & \\
\hline \multicolumn{4}{|l|}{ Developmental diagnosis } \\
\hline Intellectual disability & $29(45 \%)$ & $36(55 \%)$ & 0.169 \\
\hline Total & $45(49 \%)$ & $46(51 \%)$ & \\
\hline
\end{tabular}

NOS: not otherwise specified.

disabilities underwent colonoscopy in the four hospital-based endoscopy centers. Of these, a total of 91 patients had preparation quality available in the medical record for review, and were included in the final analysis. Mean age at the time of endoscopy was $52.6 \pm 13.4$ years, with an age range of $18-74$ years. A total of 45 patients $(49 \%)$ had adequate or good bowel preparation, while 46 patients $(51 \%)$ had inadequate or poor preparation (Table 3).

On univariate analysis, rates of adequate bowel preparation were significantly higher for individuals who had their endoscopy performed in the outpatient setting compared to those who had it in the inpatient setting $(\mathrm{P}=0.031)$. When odds ratios were calculated using the data obtained from univariate analysis, individuals who had their endoscopies performed in the outpatient setting were found to be 2.75 times more likely to have adequate bowel preparations compared to those in the inpatient setting (Table 4). Also, all patients with a diagnosis of Developmental Disorder NOS had significantly better bowel preparation based on analysis $(\mathrm{P}=0.026)$. However, the low number of cases in this subgroup $(n=5)$ yields results that may not be statistically reliable. No other factors studied, including age at the time of endoscopy, endoscopy indication and time of procedure, had any statistically significant influence on the adequacy of bowel preparation.

\section{Discussion}

Our study found that approximately half (51\%) of patients with developmental disabilities undergoing colonoscopy had inad-

Table 4. Odds Ratios for Select Factors Affecting Bowel Preparation Adequacy

\begin{tabular}{|c|c|c|c|}
\hline & Odds ratio & $95 \%$ confidence interval & P value \\
\hline \multicolumn{4}{|l|}{ Location } \\
\hline Outpatient & 2.75 & $1.14-6.62$ & $0.022 * *$ \\
\hline \multicolumn{4}{|l|}{ Indication } \\
\hline Therapeutic & 0.86 & $0.37-2.00$ & 0.731 \\
\hline \multicolumn{4}{|l|}{ Time of procedure } \\
\hline \multicolumn{4}{|l|}{ Developmental diagnosis } \\
\hline Down syndrome & 1.44 & $0.42-4.91$ & 0.563 \\
\hline Autism spectrum disorder & 0.56 & $0.17-1.88$ & 0.346 \\
\hline Intellectual disability & 1.99 & $0.78-5.03$ & 0.145 \\
\hline
\end{tabular}


equate bowel preparations, which is more than twice the current estimate for the general population. The rate of inadequate bowel preparation in colonoscopies for the general population has been noted to be approximately $23.1 \%$ [9]. The findings of our study support the information found in a previous study by Fischer et al, which examined forty individuals with intellectual disabilities and found inadequate bowel preparation in $46 \%$ of their patients [15]. We believe that our study was able to build upon this information by achieving higher statistical power based on available patient charts. Furthermore, we examined factors associated with the adequacy of bowel preparation and were able to identify differences based on the setting in which the bowel preparation was administered.

Contrary to our initial hypothesis, hospitalized patients had significantly worse bowel preparations compared to outpatients. We had originally hypothesized that hospitalized patients would have better bowel preparation since the purge could be administered in a more monitored setting, and with more intensive ancillary support. In exploring this specific aspect, a recent large study involving 3,276 colonoscopies addressed this specific issue in the general population, where researchers found no significant differences between inpatients and outpatients in terms of the rate of adequate bowel preparation in both the left colon $(76.5 \%$ versus $77.4 \%$, P $=0.578)$ and the proximal colon $(63.2 \%$ versus $65.6 \%, \mathrm{P}=$ 0.178 ) during colonoscopy [16]. One potential factor that may help explain this finding in our study is the impact of health literacy on bowel preparation [17]. A study of 764 patients who were presented with an information leaflet that outlined bowel preparatory instructions found that health literacy was a significant predictor of comprehension $(\mathrm{P}<0.001)$ [18]. Patients with developmental disabilities may not necessarily fully comprehend complex medical instructions, depending on their cognitive abilities, and may be reliant on their parents or caregivers to help navigate their medical care [19]. In addition, a potential obstacle for these patients in relation to health care access stems from communication barriers. Prior studies have found that healthcare providers report their own lack of training in regard to communicating with this specific patient population as a limitation to optimal healthcare delivery [20]. Furthermore, one study that focused on nurses caring for patients with developmental disabilities in the hospital setting found that the additional time and resources required to effectively communicate with these patients may lead nurses to avoid communication altogether, and may overlook opportunities to improve communication with the patient [21]. Considering these factors in the context of our findings, a possible obstacle for obtaining an adequate bowel preparation for patients with developmental disabilities in the hospital setting is the potential for ineffective communication between the patients receiving the purge and the healthcare personnel who administer it. In contrast, patients who receive their bowel preparation as an outpatient may experience the benefit of receiving the purge from a family member or caregiver more familiar with their complex communication challenges. In addition, these patients may also receive their purge in an environment that they are more accustomed to, such as their place of residence, rather than the unfamiliar surroundings of a hospital setting. Another potential reason would be related to the acuity or severity of the illness encountered by hospitalized patients compared to outpatients, which may affect one's ability to optimally adhere to the prescribed bowel preparation regimen.

Several limitations to our study should be recognized. Due to the retrospective nature of the study, a number of factors could not be reliably accounted for, and were thus not included in the data collection. First, cancelled procedures prior to endoscope insertion due to reports of inadequate purge intake or other factors may not have been accurately recorded in the medical record, and thus, the actual number of eligible patients may have been underestimated. Second, the information regarding the use of a nasogastric tube for purge administration for inpatients was not available. However, this information, had it been available, would theoretically lend itself towards more adequate bowel preparation in this subgroup rather than the opposite. Third, a large number (59) of reports did not have bowel preparation data available for review, and were thus excluded from the final analysis. Majority of these incomplete charts were from the period prior to the introduction of electronic endoscopic transcription software. Fourth, the level of acuity of the hospitalized patients could not be reliably obtained from the medical record and was thus excluded from data collection. Finally, information regarding the completeness of purge intake, administration regimen and type or dose of preparation was not readily available, especially for those who received this as an outpatient. It should be noted that in current clinical guidelines, the recommendations state that the higher-volume $4 \mathrm{~L}$ polyethylene glycol-electrolyte lavage solutions (PEG-ELS) has shown no superiority over lower-volume PEG preparations [8].

We recommend that further studies be performed to explore the factors relating to inadequate bowel preparation in this patient population in greater detail. A prospective study examining various factors relating to adequate bowel preparation in patients with developmental disabilities in comparison to those without these conditions in the community setting, with a standardized bowel purge administration protocol, is recommended to elucidate these factors more clearly. In the interim, we recommend that communication barriers in the context of bowel purge administration in the inpatient setting should be further assessed to achieve the optimize bowel preparation in this patient population, while providing a positive experience for these patients and their families. Another option that may be explored for these patients is to determine whether other approaches, such as 2-day bowel purge regimens, would be more beneficial in achieving an adequate bowel preparation for colonoscopy.

Although current clinical practice guidelines from the United States Multi-Society Task Force on Colorectal Cancer do address specific patient populations, including the elderly, children, pregnant patients and those with inflammatory bowel disease, patients with developmental disabilities are not specifically identified as high-risk individuals in relation to bowel preparation [8]. To further emphasize this point, a study that examined clinical guidelines from seven countries found that the majority of these guidelines failed to address special needs of individuals with developmental disabilities whenever applicable, despite the growing body of evidence illustrating the health disparities experienced by this population [22]. With 
more studies examining the unique needs of these patients prior to, during and after endoscopy, it is our hope that this group would be addressed specifically in future endoscopic guidelines.

In conclusion, individuals with developmental disabilities represent a unique population in the setting of gastrointestinal endoscopy. These patients have much higher rates of inadequate bowel preparation, more than twice as high when compared to the general population. Hospitalized patients with developmental disabilities, in particular, are more likely to have inadequate bowel preparation compared to those who receive their purge as an outpatient. Further studies are recommended to improve endoscopic practices for this patient population.

\section{Acknowledgments}

The authors would like to acknowledge the various contributions of Dr Seina Farshadsefat, Dr Abdullah Haidar, Dr Ruby Jhaj, Dr Kamran Kalim, Dr Daniyeh Khurram, Dr David Svinarich and Dr Susanna Szpunar to this study.

\section{Conflict of Interest}

The authors have no potential conflict of interest to disclose.

\section{Financial Support}

None.

\section{Presentation}

The study was presented as Bowel Preparation Adequacy in Patients with Developmental Disabilities; American College of Gastroenterology (ACG) Annual Scientific Meeting 2015 Poster Presentation. Honolulu, HI, USA. October 20, 2015 (Poster).

\section{Ethics Consent}

This study received approval of the site Institutional Review Board (IRB).

\section{References}

1. Boyle CA, Boulet S, Schieve LA, Cohen RA, Blumberg SJ, Yeargin-Allsopp M, Visser S, et al. Trends in the prevalence of developmental disabilities in US children, 1997-2008. Pediatrics. 2011;127(6):1034-1042.

2. Patja K, Iivanainen M, Vesala H, Oksanen H, Ruoppila I. Life expectancy of people with intellectual disability: a 35-year follow-up study. J Intellect Disabil Res. 2000;44(Pt 5):591-599.
3. Bittles AH, Glasson EJ. Clinical, social, and ethical implications of changing life expectancy in Down syndrome. Dev Med Child Neurol. 2004;46(4):282-286.

4. Traci MA, Seekins T, Szalda-Petree A, Ravesloot C. Assessing secondary conditions among adults with developmental disabilities: a preliminary study. Ment Retard. 2002;40(2):119-131.

5. Book L, Hart A, Black J, Feolo M, Zone JJ, Neuhausen SL. Prevalence and clinical characteristics of celiac disease in Downs syndrome in a US study. Am J Med Genet. 2001;98(1):70-74.

6. Anderson LL, Humphries K, McDermott S, Marks B, Sisirak J, Larson S. The state of the science of health and wellness for adults with intellectual and developmental disabilities. Intellect Dev Disabil. 2013;51(5):385-398.

7. Ouellette-Kuntz H, Coo H, Cobigo V, Wilton AS. Uptake of colorectal cancer screening among Ontarians with intellectual and developmental disabilities. PLoS One. 2015;10(2):1-14.

8. Johnson DA, Barkun AN, Cohen LB, Dominitz JA, Kaltenbach T, Martel M, Robertson DJ, et al. Optimizing adequacy of bowel cleansing for colonoscopy: recommendations from the US multi-society task force on colorectal cancer. Gastroenterology. 2014;147(4):903-924.

9. Harewood GC, Sharma VK, de Garmo P. Impact of colonoscopy preparation quality on detection of suspected colonic neoplasia. Gastrointest Endosc. 2003;58(1):76-79.

10. Chokshi RV, Hovis CE, Hollander T, Early DS, Wang JS. Prevalence of missed adenomas in patients with inadequate bowel preparation on screening colonoscopy. Gastrointest Endosc. 2012;75(6):1197-1203.

11. Rex DK, Imperiale TF, Latinovich DR, Bratcher LL. Impact of bowel preparation on efficiency and cost of colonoscopy. Am J Gastroenterol. 2002;97(7):1696-1700.

12. Sullivan PB. Gastrointestinal disorders in children with neurodevelopmental disabilities. Dev Disabil Res Rev. 2008;14(2):128-136.

13. Fatima H, Johnson CS, Rex DK. Patients' description of rectal effluent and quality of bowel preparation at colonoscopy. Gastrointest Endosc. 2010;71(7):1244-1252 e1242.

14. Aronchick CA, Lipshutz WH, Wright SH, Dufrayne F, Bergman G. A novel tableted purgative for colonoscopic preparation: efficacy and safety comparisons with Colyte and Fleet Phospho-Soda. Gastrointest Endosc. 2000;52(3):346-352.

15. Fischer LS, Becker A, Paraguya M, Chukwu C. Colonoscopy and colorectal cancer screening in adults with intellectual and developmental disabilities: review of a series of cases and recommendations for examination. Intellect Dev Disabil. 2012;50(5):383-390.

16. Rotondano G, Rispo A, Bottiglieri ME, De Luca L, Lamanda R, Orsini L, Bruzzese D, et al. Quality of bowel cleansing in hospitalized patients undergoing colonoscopy: A multicentre prospective regional study. Dig Liver Dis. 2015;47(8):669-674.

17. Nguyen DL, Wieland M. Risk factors predictive of poor quality preparation during average risk colonoscopy screening: the importance of health literacy. J Gastroin- 
testin Liver Dis. 2010;19(4):369-372.

18. Smith SG, von Wagner C, McGregor LM, Curtis LM, Wilson EA, Serper M, Wolf MS. The influence of health literacy on comprehension of a colonoscopy preparation information leaflet. Dis Colon Rectum. 2012;55(10):10741080.

19. Redley M, Prince E, Bateman N, Pennington M, Wood $\mathrm{N}$, Croudace T, Ring H. The involvement of parents in healthcare decisions where adult children are at risk of lacking decision-making capacity: a qualitative study of treatment decisions in epilepsy. J Intellect Disabil Res. 2013;57(6):531-538.
20. Reichard A, Turnbull HR. Perspectives of physicians, families, and case managers concerning access to health care by individuals with developmental disabilities. Ment Retard. 2004;42(3):181-194.

21. Hemsley B, Balandin S, Worrall L. Nursing the patient with complex communication needs: time as a barrier and a facilitator to successful communication in hospital. J Adv Nurs. 2012;68(1):116-126.

22. Mizen LA, Macfie ML, Findlay L, Cooper SA, Melville CA. Clinical guidelines contribute to the health inequities experienced by individuals with intellectual disabilities. Implement Sci. 2012;7:42. 